\title{
ARTICLE \\ Aeromonas hydrophila utilizes TLR4 topology for synchronous activation of MyD88 and TRIF to orchestrate anti- inflammatory responses in zebrafish
}

\author{
Nidhi Srivastava ${ }^{1}$, Asha Shelly ${ }^{1}$, Manmohan Kumar ${ }^{1}$, Archana Pant ${ }^{2,3}$, Bhabatosh Das ${ }^{2,3}$, Tanmay Majumdar ${ }^{1}$ and Shibnath Mazumder ${ }^{1}$
}

Toll-like receptor 4 (TLR4) plays a critical role in host immunity against Gram-negative bacteria. It transduces signals through two distinct TIR-domain-containing adaptors, MyD88 and TRIF, which function at the plasma membrane and endosomes, respectively. Using zebrafish Aeromonas hydrophila infection model, we demonstrate that synchronization of MyD88 and TRIF dependent pathways is critical for determining the fate of infection. Zebrafish were infected with A. hydrophila, and bacterial recovery studies suggested its effective persistence inside the host. Histopathological assessment elucidates that $A$. hydrophila did not provoke inflammatory responses in the spleen. Immunofluorescence revealed the presence of TLR4-bound $A$. hydrophila on the plasma membrane at $3 \mathrm{~h}$ post-infection (p.i.), and inside endosomes 1 day p.i. Quantitative PCR studies suggest that TLR4 activates the downstream pathway of MyD88-IRAK4 axis at early stages followed by a shift to TRIF-TRAF6 axis at late stages of infection coupled with fold increase in NFKB. Our results implicated the involvement of $\mathrm{p} 110 \delta$ isoform of $\mathrm{PI}(3)$ Kinase in this transition. Coupled to this, we noted that the TLR4-TRIF-NFKB axis prompted burgeoned secretion of anti-inflammatory cytokines. We observed that A. hydrophila inhibits endosome maturation and escapes to cytoplasm. Significant downregulation of cytosolic-NLR receptors further suggested that A. hydrophila represses pro-inflammatory responses in cytosol aiding its persistence. Our findings suggest a novel role of 'TLR4 topology' in A. hydrophila-induced pathogenesis. We propose that A. hydrophila manipulates translocation of TLR4 and migrates to endosome, where it triggers TRIF-dependent anti-inflammatory responses, interferes with endosomal maturation and escapes to cytosol. Inside the cytosol, A. hydrophila avoids detection by suppressing NLRs, facilitating its survival and ensuing pathogenesis.

Cell Death Discovery (2017) 3, 17067; doi:10.1038/cddiscovery.2017.67; published online 2 October 2017

\section{INTRODUCTION}

The sensing of microbes is mediated by innate pattern recognition receptors (PRRs), which include Toll-like receptors (TLRs). ${ }^{1}$ TLRs belong to type 1 transmembrane protein family and comprise an $\mathrm{N}$-terminal extracellular domain that recognizes pathogenassociated molecular patterns, transmembrane domain and C-terminal or intracellular Toll/interleukin-1 receptor (TIR) domain which serves as a docking site for adaptor molecules important for downstream signaling. ${ }^{2}$ Among TLR family members, TLR4 was the first identified and recognized as an important innate immune receptor. ${ }^{3,4}$ Once TLR4 binds to its cognate ligand, lipopolysaccharide of Gram-negative bacteria, it activates two distinct signaling pathways - the MyD88-dependent and TRIFdependent pathways. Signaling through these two pathways leads to expression of both pro- and anti-inflammatory cytokines and immunomodulatory molecules. ${ }^{5}$ MyD88-dependent signaling requires the sorting adaptor TIRAP/Mal at the plasma membrane, which helps in induction of NFKB and mitogen-activated protein kinase (MAPK) pathways culminating in pro-inflammatory responses. On the other hand, the MyD88-independent or TRIFdependent pathway is endosomal and initiated by the sorting adaptor TRAM. The TIRAP-MyD88 pathway transits sequentially into TRAM-TRIF signaling, though the mechanisms remain unknown. It was observed that p110 $\delta$, which is the main class I $\mathrm{PI}(3) \mathrm{K}$ isoform, 'licenses' the internalization of TLR4 from the plasma membrane to endosome. ${ }^{6}$ The transition of TLR4 from an early-acting TIRAP-MyD88 associated plasma membrane complex into a late-acting endosomal TRAM-TRIF complex is often associated with switch from pro-inflammatory to antiinflammatory responses. ${ }^{5}$

The presence of TLR4 is well reported in fish. ${ }^{7-14}$ However, the role of fish TLR4 as a receptor for LPS is contentious. Compared with mammals, fish can tolerate relatively higher concentrations of LPS. ${ }^{15}$ The lack of LBP, MD2 and CD14, essential for LPS binding to TLR4 in all fish genomes studied till date, ${ }^{16}$ clearly suggests the mechanism of LPS recognition in fish to be different from that of mammals. ${ }^{7,16,17}$ Zebrafish is known as a sentinel species to model human diseases ${ }^{18}$ as it has a fully mapped genome significantly homologous with that of humans. ${ }^{19}$ Zebrafish TLR4 has been cloned and characterized. ${ }^{20,21}$ It exists as two TLR4 paralogs TLR4a and TLR4 ${ }^{7}$ - which show sequence difference in the extracellular protein domain. ${ }^{17}$ There are reports suggesting that zebrafish TLR4 fails to recognize LPS and negatively regulates MyD88-NFKB axis. $^{7}$

Aeromonas hydrophila, Gram-negative rod-shaped bacterium, shows wide host tropism. In fish, it causes aeromonad septicemia, ${ }^{22,23}$ though the pathogenesis is not well understood. The bacteria expresses diverse array of virulence factors which together with environmental cues manifest pathogenic effects in diverse hosts. ${ }^{24,25}$ In fish, spleen is an important immune organ

${ }^{1}$ Immunobiology Laboratory, Department of Zoology, University of Delhi, Delhi, India; ${ }^{2}$ Translational Health Science and Technology Institute, NCR Biotech Science Cluster, Faridabad, India and ${ }^{3}$ School of Life Sciences, Manipal University, Manipal, Karnataka, India.

Correspondence: N Srivastava (nidhibhu@yahoo.com) or S Mazumder (shibnath1@yahoo.co.in)

Received 14 August 2017; accepted 18 August 2017; Edited by A Rufini 
that participates in mounting both innate and adaptive immune responses and helps in the clearance of microbial pathogens in fish. ${ }^{26,27}$ Although, there are several reports documenting the active involvement of several fish immune molecules during A. hydrophila infections, ${ }^{28-32}$ information regarding the role of TLR4 and the associated downstream signaling pathway in A. hydrophila pathogenesis still remains unexplored. To look into this we have used the zebrafish A. hydrophila infection model and report that the spatial distribution of TLR4 (plasma membrane vs endosomal) governs dichotomous TLR4 signaling, thereby influencing microbial pathogenesis.

\section{RESULTS}

Clinical signs of $A$. hydrophila infection in zebrafish are dose dependent

At the onset we aimed to determine the LD50 dose of the $A$. hydrophila isolate in zebrafish. Zebrafish were infected with three different doses of live $A$. hydrophila and mortality along with phenotypic changes monitored. Infection with $1 \times 10^{9}$ CFU led to $100 \%$ mortality within 1 day post-infection (p.i.) and the infected fish did not show any signs of disease manifestations. Infection with $1 \times 10^{7}$ CFU led to $78 \%$ mortality within 4 days of infection (Figure 1) and the infected fish showed severe petechial hemorrhages on the abdomen as well as at the base of the fins. The fish stopped feeding, remained listless at the bottom of the tanks and rarely came to surface for gulping air. Infection with $1 \times 10^{6} \mathrm{CFU}$ failed to induce any phenotypic change and fish death (Figure 1). Based on the mortality results, the 4-day LD50 dose of $A$. hydrophila was calculated to be $8 \times 10^{6} \mathrm{CFU}$. We selected $6 \times 10^{6} \mathrm{CFU}$ as the infection dose which caused $7 \%$ mortality over a period of 4 days (Figure 1) and the infected fish exhibited peritonitis and localized hemorrhagic lesions. Control and sham-injected fish did not show any mortality. All the dead fishes were homogenized, plated on Aeromonas selective medium plates and the presence of A. hydrophila colonies were noted (data not shown).

Histological analysis elucidates absence of inflammation in zebrafish spleen

The next step was studying A. hydrophila-induced histopathological alterations. For this we selected spleen, an important

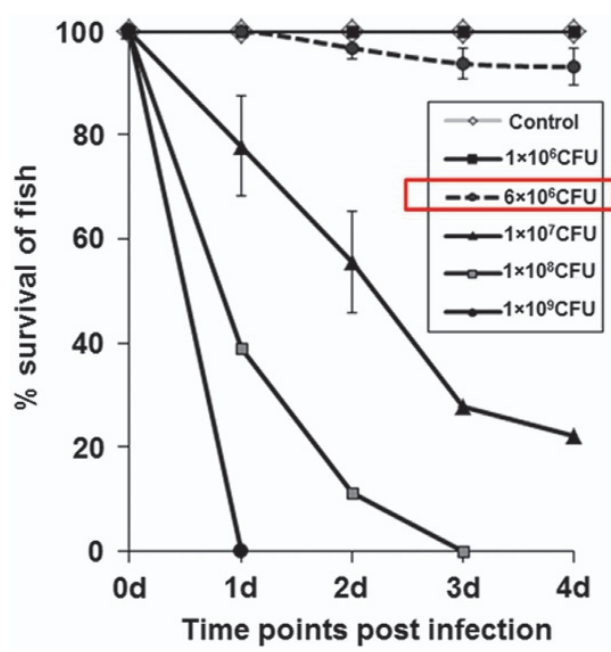

Figure 1. A. hydrophila induced dose-dependent mortality in zebrafish. Zebrafish were injected i.p. with different CFU of $A$. hydrophila and the mortality was recorded at indicated time points. Data represent the mean value of three independent experiments ( $n=10$ /experiment) and the error bars represent standard deviations. $d$, days. immune organ in fish. The parenchyma of spleen comprises of two major functional zones - the hematogenous red pulp and the lymphoid white pulp (Figure 2a). The red pulp houses the erythrocytes and thrombocytes while the white pulp possesses the lymphatic tissue. The presence of ellipsoids is often noted at the terminals of splenic arterioles (Figure 2a). In infected zebrafish, a significant expansion of the red pulp area was observed (Figure 2b). Other cellular alterations like hypertrophied cells, hyperplasia, lymphocyte infiltration and edematous swelling were not evident in the parenchyma of infected zebrafish spleen. These results suggest that $A$. hydrophila does not provoke proinflammatory changes in splenic parenchyma.

\section{TLR4 signaling is critical in A. hydrophila pathogenesis}

The role of fish TLR4 is well implicated in immunity to Gramnegative bacteria. ${ }^{33}$ We were interested to study TLR4 signaling in zebrafish spleen following $A$. hydrophila infection. To this end, zebrafish were infected with $6 \times 10^{6}$ CFU of $A$. hydrophila, spleen removed and TLR4 expression monitored by qPCR at indicated time points. We observed maximum TLR4 expression at $3 \mathrm{~h}$ p.i., thereafter the levels though declined remained significantly high till 1 day p.i. $(P<0.05)$ (Figure 3a). These results suggested the involvement of TLR4 in A. hydrophila pathogenesis in zebrafish.

\section{A. hydrophila triggers MyD88 and TRIF in a sequential manner}

TLR4 signaling is mediated by two distinct adapter molecules, MyD88 and TRIF., ${ }^{5,6}$ In absence of prior information, we aimed to decipher the relative involvement of the two adapter molecules during the course of $A$. hydrophila pathogenesis in fish. It is evident from Figure $3 b$ that significant MyD88 $(P<0.05)$ expression occurred in spleen only at the earlier time points, that is, at 3 and $6 \mathrm{~h}$ p.i. On the contrary, significant expression of TRIF was noted at later time points with the maximum fold increase recorded in 2 days post-infected spleens (Figure 3b). Together, these results for the first time demonstrated differential TLR4 signaling in course of $A$. hydrophila pathogenesis wherein MyD88 pathway is involved at the early stages and TRIF pathway at later stages of infection.

\section{TLR4 signaling is divaricated in zebrafish spleen}

Our next aim was to explore the downstream signaling cascade involved in $A$. hydrophila pathogenesis. We monitored the expression of IRAK4 (IL-1 receptor-associated kinase), TRAF6 and TRAF3 (TNF receptor-associated factors). It was observed that though IRAK4 expression started increasing from $6 \mathrm{~h}$ p.i. $(P=0.063)$, significant increase was recorded at $12 \mathrm{~h}$ p.i. $(P<0.05)$, thereafter it declined and reached basal levels at 1 day p.i. Our results suggested significant upregulation of RIP1 $(P<0.05)$ and TRAF6 $(P<0.05)$ at 1 and 2 days p.i. (Figure $3 c)$. The expression of TRAF3 remained undetected throughout the experiment. We followed this by studying the fold change in NF $K B$ and it is evident from Figure $3 c$ that its expression started increasing from $12 \mathrm{~h}$ p.i. $(P=0.144)$ with a significant fold change recorded $(P<0.05)$ in 1 and 2 days infected spleen. To this we infer that consequent to $A$. hydrophila infection two signaling pathways are initiated in zebrafish; at early stages TLR4-MyD88IRAK4-TRAF6-NFKB is functional and at the later stages signaling is through the TLR4-TRIF-RIP1-TRAF6-NFKB axis.

\section{A. hydrophila pathogenesis induces anti-inflammatory response in} zebrafish

The dichotomy in TLR4 downstream signaling pathways prompted us to study cytokine expression in course of $A$. hydrophila infection. Zebrafish were infected with $A$. hydrophila and the expression of key pro- and anti-inflammatory cytokines studied. We failed to detect the expression of pro-inflammatory cytokines 

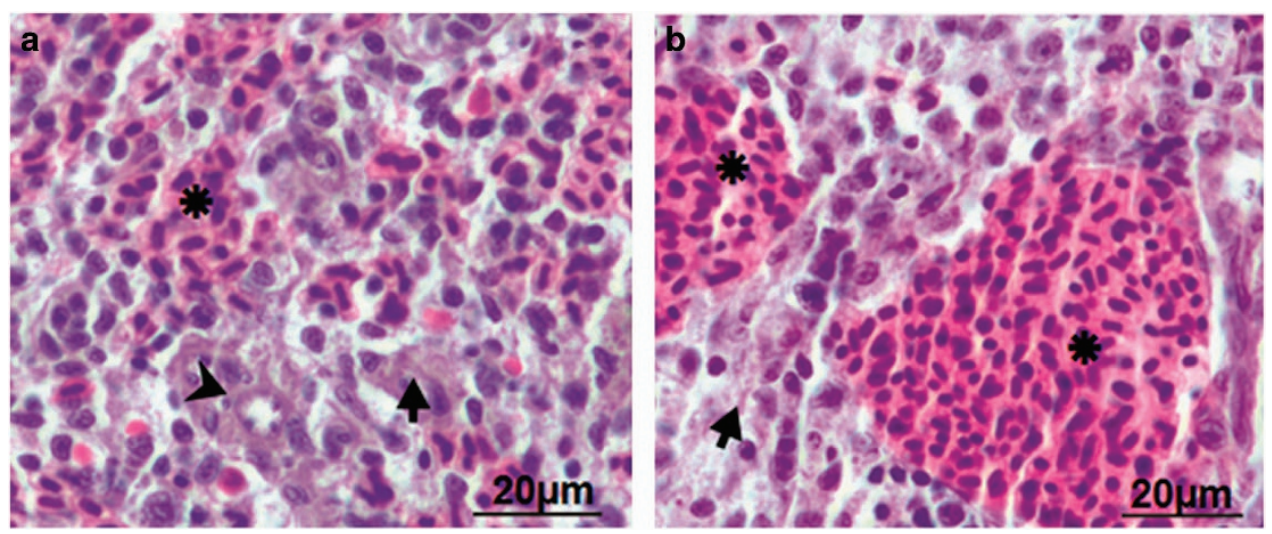

Figure 2. Histological analysis elucidated absence of inflammation in zebrafish spleen. Spleen was removed from control and infected zebrafish and the parenchyma of both was compared $(\times 40)$. (a) Control zebrafish spleen comprises hematogenous red pulp (asterisk), lymphoid white pulp (arrow) and ellipsoid (arrow head). (b) A. hydrophila-infected fish spleen revealed significant expansion of the red pulp area.
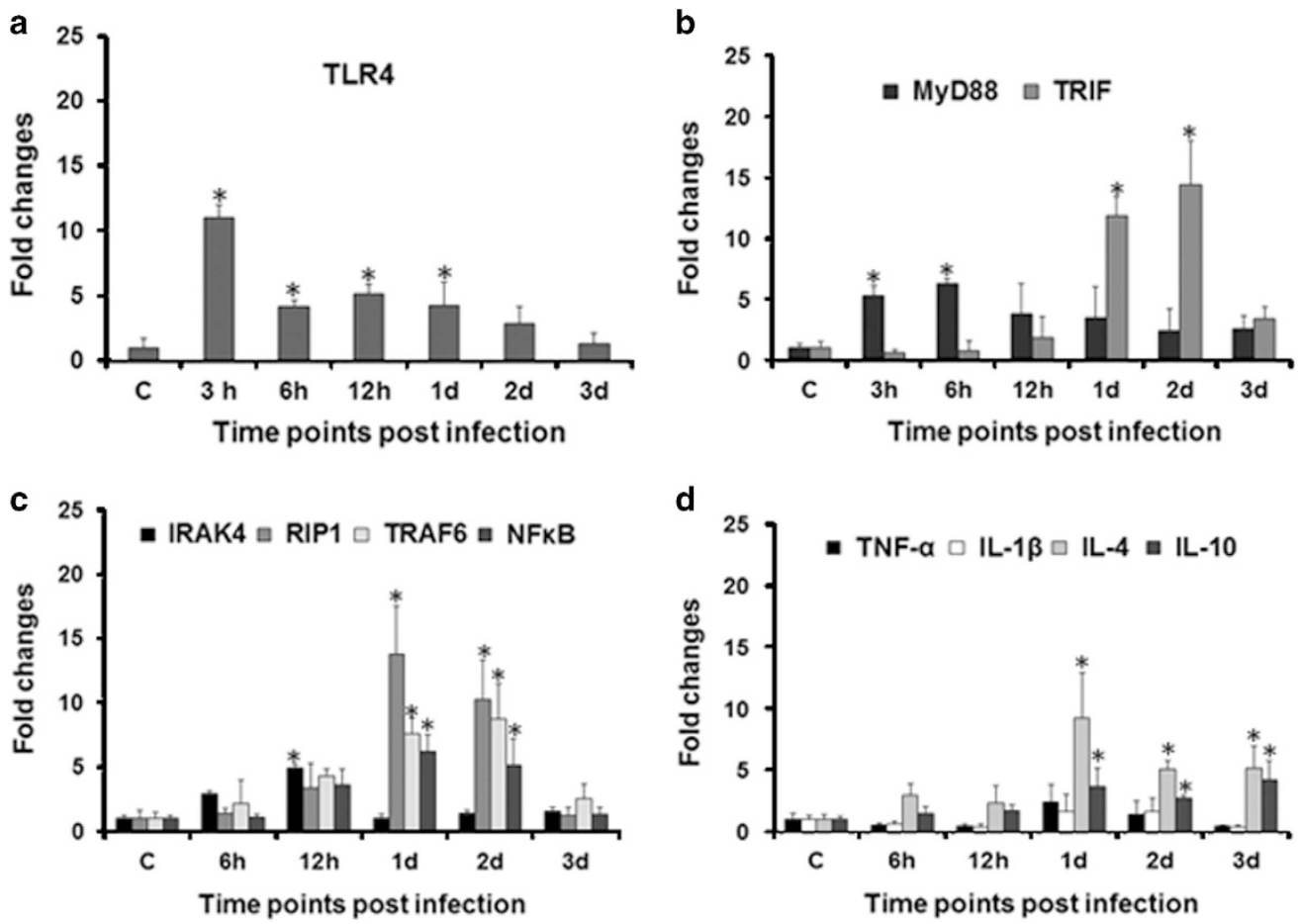

Figure 3. Expression studies of TLR4 signaling pathway-related genes. Spleen was removed from control and infected zebrafish at indicated time points, total RNA isolated, cDNA prepared which was used for qPCR analysis of (a) TLR4; (b) MyD88, TRIF; (c) IRAK4, RIP1, TRAF6, NFKB; (d) TNF- $\alpha$, IL-1 $\beta$, IL-4, IL-10. Each bar represent the mean of three independent experiments ( $n=5$ /experiment) and the error bars represent the standard deviations. $\mathrm{C}$, control; $\mathrm{h}$, hours; $\mathrm{d}$, days; and asterisks ' $*$ ' on bars indicate significant difference from control $(P<0.05)$.

IFN- $\gamma$ and IL-12 at all the time points studied. This was followed by studying the kinetics of two other pro-inflammatory cytokines TNF- $\alpha$ and IL- $1 \beta$ and we did not observe any noticeable increase in their expression levels (Figure $3 \mathrm{~d}$ ). Contrastingly, the expression of IL-4 and IL-10 was found to be upregulated throughout the experiment with a significant fold increase $(P<0.05)$ recorded from 1 day onwards in infected spleen. Collectively, these results suggested that $A$. hydrophila infection primarily induces antiinflammatory responses in zebrafish.

\section{A. hydrophila persists inside zebrafish}

The next step was to correlate the anti-inflammatory response with bacterial persistence. Zebrafish were infected with live A. hydrophila, killed at indicated time points, head and fins removed, homogenized, plated on Aeromonas selective medium and bacterial load quantified by counting CFU. We observed significant increase in the bacterial load at 1 day p.i., which remained almost similar at 2 days. Thereafter, though it declined, the bacterial number never reached basal level (Figure 4). Control fish did not exhibit any bacterial colony at any time intervals.

\section{A. hydrophila gets endocytosed after being recognized at the} membrane

The time kinetics of MyD88 as well as the TRIF-dependent pathway prompted us to explore the reason behind the differential activation of both the pathways. To study this, zebrafish were infected with mCherry-tagged $A$. hydrophila and bacterial movement inside splenic macrophages monitored at 
indicated time intervals using the early endosomal marker, EEA1 (green fluorescence). It is evident from our immunofluorescence studies that at the early stages of infection $(3 \mathrm{~h})$, the bacteria remain localized on the membrane of macrophages (red fluorescence) (Figure 5a). The yellow fluorescence (merged) observed in macrophages collected 1 day p.i. suggested the presence of bacteria in the early endosomes (Figure 5a). Macrophages obtained from 2 days post-infected spleen did not display yellow fluorescence but there were several red fluorescent dots in the cytoplasm (Figure 5a) indicating the presence of $A$. hydrophila

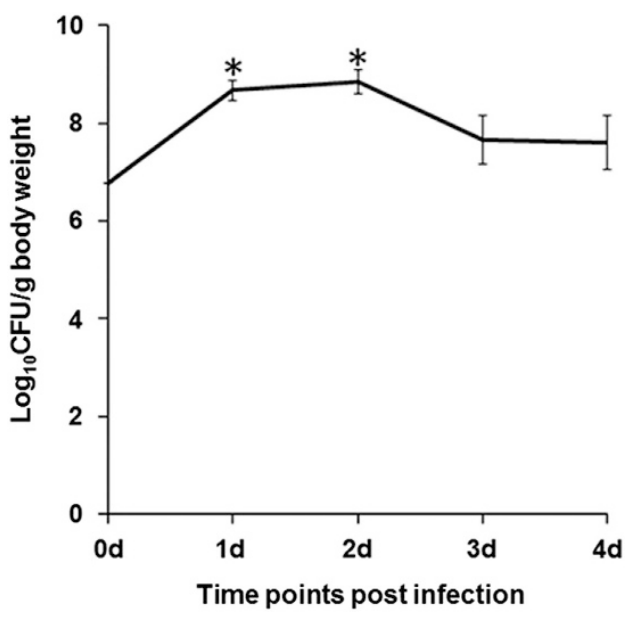

Figure 4. A. hydrophila persists in infected zebrafish. Zebrafish were infected with $A$. hydrophila $\left(6 \times 10^{6} \mathrm{CFU}\right)$ and the bacterial load was studied in the whole fish by counting CFU at the indicated time points. Data represent mean value of three independent experiments ( $n=5$ /experiment) and the error bars represent standard deviations. $d$, days and asterisks ' $*$ ' indicate significant difference from CFU at the time of infection $(P<0.05)$. in the cytoplasm of infected zebrafish splenic macrophages (zSPM).

To substantiate bacterial movement through endosomes, we monitored the expression of TLR9, an endosomal TLR. In corroboration to our immunofluorescence study we observed significant upregulation in TLR9 expression in 1 day infected spleen $(P<0.05)$ followed by sudden decline from 2 days p.i. $(P<0.05)$ (Figure 5b).

\section{A. hydrophila escapes from the early endosomes}

The immunofluorescence results encouraged us to study A. hydrophila movement after egressing early endosomes. The expression of RAB7, a bonafide marker for the late endosomes, was checked, which revealed significant upregulation at 1 day p.i. (Figure 5b). Thereafter RAB7 expression declined reaching basal levels in 2 and 3 days post-infected spleen, suggesting limited transition from early to late endosome formation in A. hydrophilainfected macrophages. The presence of $A$. hydrophila in the cytoplasm prompted us to check the expression pattern of cytosolic sensor NOD2 at the later time points when the bacterium moved to the cytosol. We observed significant downregulation of NOD2 expression $(P<0.05)$ in 2 and 3 days post-infected spleen (Figure $5 \mathrm{C}$ ), suggesting its inhibition to be a survival strategy for $A$. hydrophila inside the macrophages.

TLR4 internalization is supported by $\mathrm{p} 110 \delta$ isoform of the kinase $\mathrm{PI}(3) \mathrm{K}$

The dichotomy in TLR4 signaling prompted us to study its localization in infected zSPM. The ZSPM were permeabilized and stained with TRITC-conjugated anti-TLR4 antibody and FITCconjugated anti-EEA1 at indicated time points and observed under a fluorescence microscope. Red fluorescence observed in control zSPM and at the early stage of infection ( $3 \mathrm{~h}$ ) advocates that initially TLR4s remain at the membrane (Figure 6a) but at 1 day p.i. the appearance of yellow fluorescence indicates that they get internalized into the endosome at this time point (Figure 6a). The internalization of TLR4 is mediated through p110 $\delta$ a
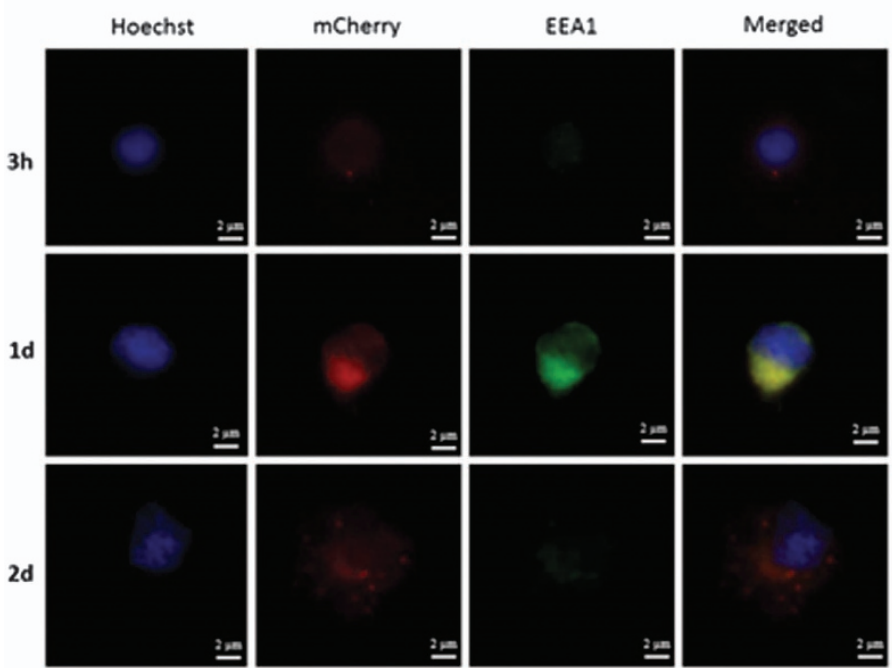
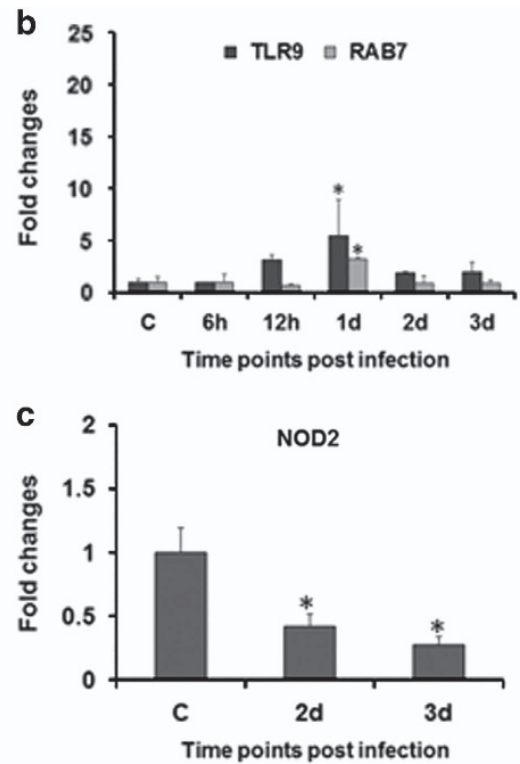

Figure 5. A. hydrophila trafficking in zebrafish. (a) Zebrafish were infected with mCherry-tagged A. hydrophila. Bacterial localization was revealed by immunofluorescence staining of zSPM using Hoechst 33342 and EEA1 at indicated time points ( $\times 100)$. (b, c) Expression of TLR9, RAB7 and NOD2 was determined from spleen of control and infected fishes. Each bar represent the mean of three independent experiments ( $n=5$ /experiment) and the error bars represent the standard deviations. C, control; d, days; h, hours; zSPM, zebrafish splenic macrophages; and asterisks ' $*$ ' on bars indicate significant difference from control $(P<0.05)$. 
a
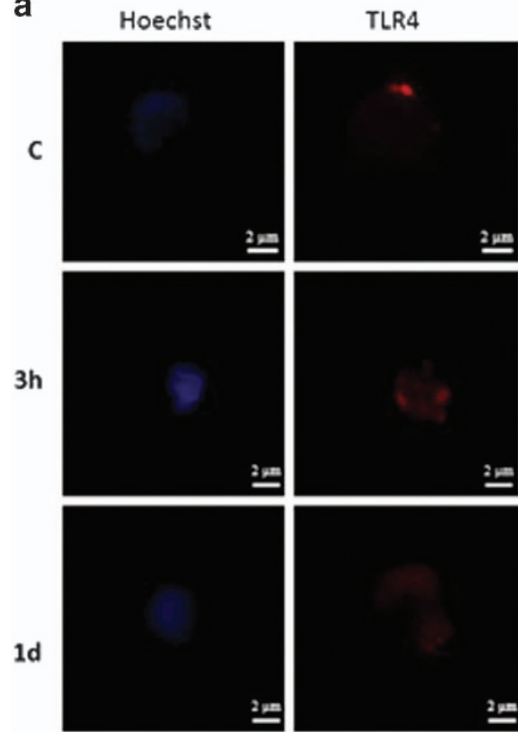
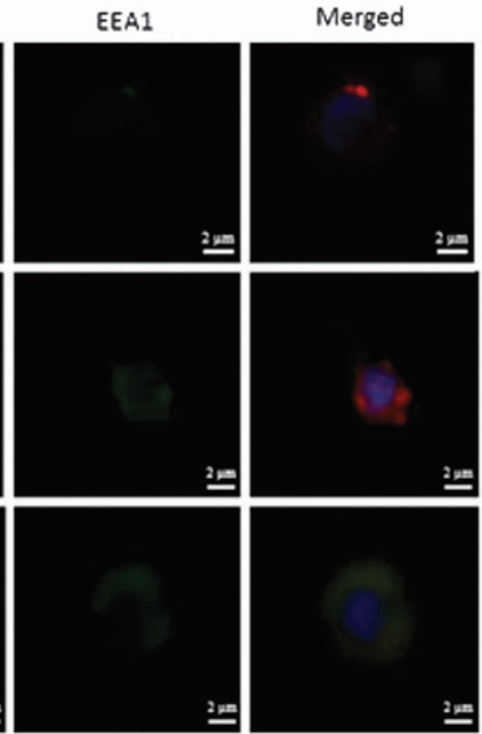

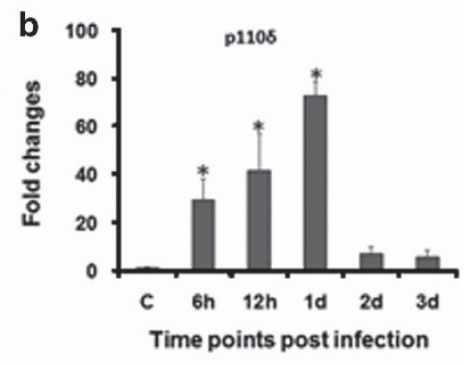

Figure 6. Subcellular distribution of TLR4. (a) zSPM were isolated from control and infected fish, stained with Hoechst 33342 , TLR4 and EEA1 antibody for TLR4 internalization study $(\times 100)$. (b) Expression of p110 $\delta$ was determined from spleen of control and infected fishes. Each bar represent the mean of three independent experiments $(n=5$ /experiment) and the error bars represent the standard deviations. $C$, control; $\mathrm{d}$, days; h, hours; zSPM, zebrafish splenic macrophages and asterisks ' $*$ ' on bars indicate significant difference from control $(P<0.05)$.

isoform of the kinase $\mathrm{PI}(3) \mathrm{K}^{6}$ Our $\mathrm{qPCR}$ data support this statement as we noted maximum upregulation of $110 \delta$ mRNA expression in zSPM collected from 1 day post-infected spleen $(P<0.05)$ followed by its decline from 2 days onwards in the infected spleen (Figure 6b).

\section{DISCUSSION}

A. hydrophila-induced pathogenesis in fish is not well understood. Though there are reports suggesting TLR4 imperative for innate recognition and regulating $A$. hydrophila pathogenesis in fish, ${ }^{8,11,33-35}$ the underlying mechanisms remain unknown. In mammals, the temporal distribution of TLR4 between plasma membrane and endosome facilitates its interaction with the adaptor molecules MyD88 and TRIF, respectively, dictating the outcome of innate immune signaling. We were interested to study how fish TLR4 co-ordinates the activation of the two distinct pathways ensuing $A$. hydrophila pathogenesis.

We observed maximum TLR4 expression at the earlier stages of infection, thereafter the levels though declined remained significant till 1 day p.i. in the spleen. The early expression of TLR4 correlates with its sentinel role as PRR during microbial infections. At early stages, plasma membrane-associated TLR4 engages MyD88 to trigger the production of pro-inflammatory cytokines. ${ }^{5}$ We noted significant expression of MyD88 at initial stages of A. hydrophila infection in zebrafish. TLR4 also activates TRIF signaling in endosome after relocation from the plasma membrane. $^{36-38}$ In accordance to this, we observed MyD88 expression replaced by TRIF at later stages of infection. Our immunofluorescence results revealed TLR4-associated bacteria on the plasma membrane at $3 \mathrm{~h}$, when MyD88 expression was highest and inside the early endosomes at 1 day p.i., the time point when TRIF expression was significant. TLR9 is an endosomal marker and its expression is triggered by unmethylated bacterial CpG-containing DNA motifs. ${ }^{39}$ We reasoned that the presence of A. hydrophila in endosome would trigger TLR9 expression. Elevated TLR9 mRNA expression in 1 day post-infected spleen further confirmed the presence of $A$. hydrophila inside the endosome. Our results corroborate earlier findings of Reyes-
Becerril et al. (2011) on upregulation in TLR9 expression in spleen, intestine and headkidney of Sparus aurata following experimental infection with $A$. hydrophila. Based on these findings, we propose that $A$. hydrophila exploits TLR4 topology to bypass MyD88mediated pro-inflammatory responses and survive in host.

We were interested to understand the translocation of TLR4 to endosome. It has been observed that the $\mathrm{PI}(3) \mathrm{K}$ isoform $\mathrm{p} 110 \delta$ directs translocation of TLR4 from the plasma membrane to endosome. ${ }^{6}$ We studied $\mathrm{p} 110 \delta$-mRNA expression and observed its gradual upregulation which was significant at 1 day p.i., suggesting the importance of $\mathrm{p} 110 \delta$ in the endosomal translocation of TLR4. Our observations corroborate earlier findings in mammals, ${ }^{6}$ suggesting this functional attribute of $\mathrm{p} 110 \delta$ to be evolutionarily conserved.

The next step was to study the involvement of downstream molecules IRAK4, RIP1 and TRAF6 in TLR4-associated signaling consequent to $A$. hydrophila infection. Activated MyD88 recruits members of the IRAK family, ${ }^{40}$ and in accordance to this we observed upregulated IRAK4 expression only at the earlier time points that coincides with increased MyD88 expression in infected spleen. This suggested activation of MyD88-IRAK4 axis, an early event in TLR4 signaling during $A$. hydrophila pathogenesis in zebrafish. RIP1, member of the serine-threonine protein kinase family related to IL-1 receptor-associated kinases, determines cellular fate following pathogen recognition. ${ }^{41}$ It serves as a bridge linking MyD88 and TRIF pathways through the common adaptor TRAF6. We observed significant upregulation of RIP1 at 1 day p.i. overlapping the expression of TRIF in the infected zebrafish spleen. RIP1 and IRAKs activate TRAF6, which is an adaptor shared by both MyD88 as well as TRIF. ${ }^{42}$ TRAF6 catalyzes the activation and translocation of freed NFKB to the nucleus. ${ }^{43}$ We observed significant upregulation in TRAF6 at 1 and 2 days p.i., suggesting its leading role in TRIF-mediated pathway in $A$. hydrophila pathogenesis. Upregulation of IRAK4 and TRAF6 has been reported in fish against several bacteria including A. hydrophila. ${ }^{30,44,45}$

TRAF3, the other member of TRAF family, is recruited to TRIF prompting the release of type I interferon $\alpha / \beta{ }^{46}$ It has recently been observed that Type I interferons play key role in the 
regulation of immune and tissue homeostasis upon bacterial insult which may have beneficial or detrimental consequences for the host. ${ }^{47}$ We checked for the expression of TRAF3 and failed to get any induction of this gene, suggesting no-role of this pathway in A. hydrophila pathogenesis in zebrafish.

NFKB is activated by both MyD88- and TRIF-dependent pathways. ${ }^{48}$ The activation of RIP1 is essential for the induction of NFKB via the TLR4-TRIF axis. ${ }^{49,50}$ We observed significant expression of RIP1 at later stages of infection overlapping the expression of TRIF and NFKB, suggesting the importance of TRIFinduced $\mathrm{NFKB}$ activation in $A$. hydrophila pathogenesis in zebrafish. NFKB promotes the production of both pro- and antiinflammatory responses affecting the outcome of immune responses and disease prognosis. ${ }^{51}$ Our histological studies suggested that $A$. hydrophila did not induce profound inflammatory changes in the spleen. In line with this, QPCR results revealed that $A$. hydrophila infection led to a significant fold increase in expression of several anti-inflammatory cytokine genes. The expression of pro-inflammatory cytokine genes like TNF- $a$ and IL- $1 \beta$ remained similar to that of controls, while we failed to detect the expression of IFN- $\gamma$ and IL-12, indicating $A$. hydrophila induces robust anti-inflammatory responses in the spleen of zebrafish. Proinflammatory response enables the host to eliminate offending pathogens ${ }^{52}$ while anti-inflammatory responses promote pathogen survival inside the host. ${ }^{53}$ We hypothesized that the antiinflammatory milieu helps $A$. hydrophila to persist inside zebrafish and cause pathogenesis. To test this, zebrafish were infected with A. hydrophila and bacterial colonization studies showed the bacteria indeed persisted in the infected fish. Our observation of increase in red pulp area in the histopathological assessment also coincides with our cytokine pattern of expression as the activation of platelets leads to the production of IL-10. ${ }^{54}$ Based on these observations we propose that $A$. hydrophila selectively activates the TRIF- NFKB axis to produce anti-inflammatory cytokines minimizing collateral tissue damage and facilitating its survival and pathogenesis in zebrafish. Contrary to our results there are also reports on enhanced expression of pro-inflammatory cytokines in different fish tissues consequent to A. hydrophila. ${ }^{28-30}$

Our study suggested $A$. hydrophila escapes from endosome to cytoplasm of splenic macrophages 2 days p.i. The concomitant decline in TLR9 expression further supports bacterial egression from endosomes. ${ }^{55}$ Endosomes fuse with lysosome, facilitating the degradation of internalized bacteria. Thus endosomal escape is a ploy for pathogens to avoid lysosomal degradation and replicate in the host cytosol. ${ }^{56,57}$ RAB7 expression is frequented with the maturation of late endosomes that fuse with lysosomes. ${ }^{58} \mathrm{We}$ observed downregulated RAB7 in 2 days post-infected spleen, suggesting $A$. hydrophila manipulates endosomal maturation to prevent endosome-lysosome fusion and use cytosol as the niche for replication and inducing pathogenesis. To the best of our knowledge, this is the first report on endosomal escape of A. hydrophila. How $A$. hydrophila prevents terminal endosome maturation and escapes to cytosol is not clear and we are trying to identify virulence factors involved in the process.

The presence of bacteria in cytosol is detected by cytosolic NOD-like receptors (NLR). NOD1 and NOD2 are important NLRs that recognize peptidoglycan present on Gram-negative bacteria to activate NFKB-mediated pro-inflammatory responses. ${ }^{59}$ Although the presence of both NOD1 and NOD2 has been reported in fish, little is known about their role in regulating fish immune responses. ${ }^{60}$ As NOD2 is a macrophage-specific protein, ${ }^{61}$ we studied NOD2 expression in the spleen from 2 days p.i., which corresponds the time when the bacteria were detected in cytosol. The significant inhibition in NOD2 expression implicated A. hydrophila actively represses NOD2 expression from activating the pro-inflammatory machinery and survives in cytosol causing pathology.
How A. hydrophila survives and induce chronic pathology and fish death is not well known. We propose that $A$. hydrophila enters the host cell via TLR4, thereafter using receptor topology it migrates to endosome escaping pro-inflammatory responses. Therein, it induces anti-inflammatory responses, interferes with terminal endosomal maturation and escapes to cytosol. In the cytosol it represses NOD2 creating a favorable niche for survival and disease prognosis.

\section{MATERIALS AND METHODS}

Zebrafish care and maintenance

Healthy zebrafish $(0.47 \pm 0.09 \mathrm{~g})$ were purchased locally (Aquazona Exports, Delhi, India) and maintained in $20 \mathrm{I}$ glass aquarium under natural photoperiod at controlled room temperature $\left(25 \pm 2{ }^{\circ} \mathrm{C}\right)$. Fish were fed daily with earthworm fish flake food (Tetra bits) and acclimatized for 15 days before infection. Zebrafish maintenance was done according to the guidelines set forth by the Animal Ethics Committee of Government of India and University of Delhi (DU/ZOOL/IAEC-R/2013/32).

\section{mCherry tagging of $A$. hydrophila}

A. hydrophila (strain no. 500297, NICED, India) was used for the study. The bacterial growth conditions and methods to confirm the pathogenicity of the strain have been described earlier. ${ }^{31,32}$ The integrative expression vector pBD64 containing mCherry-encoding gene was introduced into $A$. hydrophila by conjugation. Conjugation was performed between the recipient bacteria, $A$. hydrophila and the donor bacteria $E$. coli strain $\beta-2163$ (DAP auxotroph). Briefly, freshly grown donor and recipient cultures were mixed (1:2 ratio) thoroughly, centrifuged $(3300 \times 9 ; 2 \mathrm{~min})$ and suspended in fresh Luria broth (LB) supplemented with $0.3 \mathrm{mM}$ DAP. The bacterial mixture were incubated overnight at $37^{\circ} \mathrm{C}$ on Luria agar plates supplemented with $0.3 \mathrm{mM}$ DAP. The mating mixture were re-suspended in $2 \mathrm{ml}$ of sterile LB with appropriate antibiotics, serially diluted and plated on selection plates containing arabinose $(0.1 \%)$, ampicillin and zeocin (resistance for recipient and donor strains, respectively) and incubated overnight at $37^{\circ} \mathrm{C}$. The conjugants grown in the presence of both antibiotics were selected for further studies. The transconjugants were confirmed by wide-field microscopy for mCherry expression. These transconjugants did not show any significant difference in virulence (data not shown).

\section{Bacterial colonization}

Zebrafish (10 fish per group) were injected intraperitoneally (i.p.) with different numbers of $A$. hydrophila $\left(1 \times 10^{6}-1 \times 10^{9}\right.$ CFU in $25 \mu \mathrm{l} 0.6 \%$ saline). Fish mortality was recorded for 4 days and LD50 dose was calculated using the graphical method. A sublethal dose causing $7 \%$ mortality $\left(6 \times 10^{6} \mathrm{CFU}\right)$ was selected for the further studies. The number of bacteria injected was determined retrospectively by plating on Aeromonas selective media supplemented with $100 \mathrm{gm} / \mathrm{l}$ ampicillin (HiMedia, Mumbai, India). Control zebrafish were injected with $0.6 \%$ saline.

The $A$. hydrophila isolate used in this study is sensitive to chloramphenicol and resistant to ampicillin. ${ }^{31}$ Zebrafish were maintained in chloramphenicol treated water for 7 days and transferred to fresh water and kept for 15 days to remove the residual antibiotics. Fish were randomly sampled, killed and plated on Aeromonas selective medium supplemented with ampicillin to detect the presence of residual A. hydrophila. The absence of bacterial colonies suggested the fish to be A. hydrophila free. Subsequently, these fish were infected with A. hydrophila and at indicated time points p.i. killed, homogenized, plated on selective media and incubated at $30^{\circ} \mathrm{C}$ for $24 \mathrm{~h}$ for enumerating the CFU. The number of colonies were counted and expressed as $\log _{10} \mathrm{CFU} / \mathrm{g}$ body weight.

\section{Histopathology of spleen}

Zebrafish uninfected and infected with $A$. hydrophila were cold anesthetized, ${ }^{62}$ spleen removed at indicated time points, washed and fixed in aq. Bouin's fluid. The fishes were killed after sampling. The fixed tissues were dehydrated in an ethanol series of ascending concentrations, cleared in cedar wood oil and embedded in paraffin wax (m.p. 58-60 ${ }^{\circ} \mathrm{C}$ ). Serial sections ( $6 \mu \mathrm{m}$; Spencer microtome; Medimeas, Haryana, India) were mounted on ethanol-cleaned glass slides, kept at $37^{\circ} \mathrm{C}$ overnight, 
Table 1. List of real-time primers used for indicated genes of zebrafish

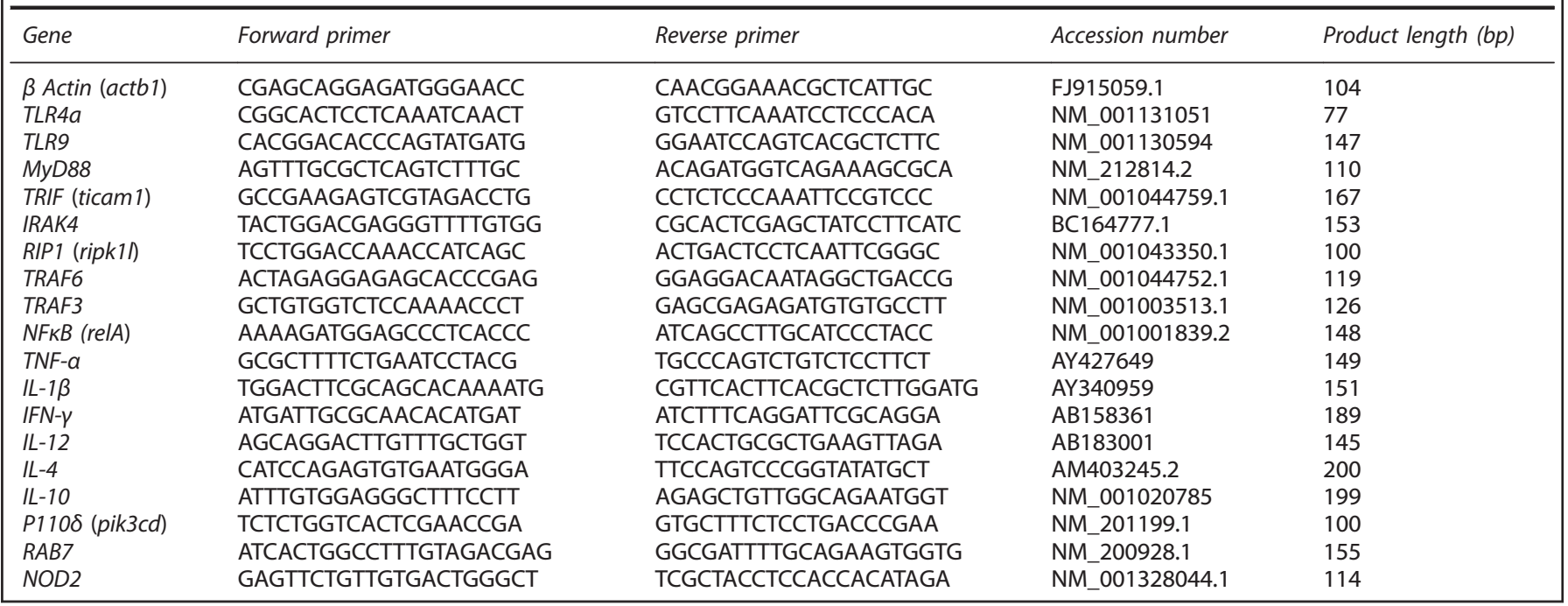

de-parafinized in xylene, hydrated in a descending ethanol series and stained with Ehrlich hematoxylin and eosin. The stained sections were dehydrated in an ascending ethanol series, cleared in xylene, then mounted in DPX and viewed under a light microscope ( $\times 40$, Nikon Eclipse 400; Nikon Instech Co. Ltd., Kangawa, Japan).

\section{RNA extraction and CDNA synthesis}

Spleen was removed from control and A. hydrophila-infected fishes at indicated time points and total RNA extracted using TRI Reagent (SigmaAldrich Corp., Darmstadt, Germany). After determining the purity and concentration, the RNA samples were incubated in $1 \mu \mathrm{l}$ of reaction buffer containing $\mathrm{MgCl}_{2}(20 \mathrm{mM})$ and $1 \mu \mathrm{l}$ of RNase free DNase I at $37^{\circ} \mathrm{C}$ for $30 \mathrm{~min}$ to eliminate genomic DNA contamination. The reaction was stopped using $1 \mu \mathrm{l}$ of EDTA $\left(50 \mathrm{mM}\right.$ ) at $65^{\circ} \mathrm{C}$ for $10 \mathrm{~min}$ and RNA reverse transcribed using the Revert Aid First Strand CDNA Synthesis Kit (Thermo Fisher Scientific, Waltham, MA, USA). The cDNA was diluted (1:100) in nuclease-free water and stored at $-20^{\circ} \mathrm{C}$ for further use.

Real-time quantitative PCR

The PCR mixture (total volume $6 \mu \mathrm{l}$ ) contained $3 \mu \mathrm{l}$ of SYBR AmpliTaq Gold DNA Polymerase (ABI), $1 \mu \mathrm{l}$ of CDNA, forward and reverse primers $(0.2 \mu \mathrm{l}$ each) and DEPC water $(1.6 \mu \mathrm{l})$. The primers used for qRT-PCR are listed in Table 1. The comparative $C t(\Delta \Delta C t)$ method was used to evaluate the expression of candidate genes using Real-Time PCR (ABI ViiA; Applied Biosystems, Foster City, CA, USA). $\beta$-Actin was used as the internal calibrator to calculate the expression of the candidate genes. The fold change in expression was used as a relative measure of gene expression.

\section{Isolation of zSPM}

Zebrafish were infected (i.p.) with or without mCherry-tagged A. hydrophila $\left(6 \times 10^{6} \mathrm{CFU}\right)$, spleen removed at indicated time points and ZSPM isolated. Briefly, spleens were placed in RPMI-1640 (Gibco/Life Technologies, Carlsbad, CA, USA), single-cell suspensions of total splenic cells were prepared using nylon mesh, washed and incubated for adherence in a Petri plate in a $\mathrm{CO}_{2}$ incubator $\left(5 \% \mathrm{CO}_{2} ; 4 \mathrm{~h} ; 30{ }^{\circ} \mathrm{C}\right)$. After incubation, nonadherent cells were removed and adherent cells were collected in a $15 \mathrm{ml}$ centrifuge tube using chilled RPMI-1640. Cells were then washed at $400 \times g$ $\left(4{ }^{\circ} \mathrm{C} ; 10 \mathrm{~min}\right.$ ) and re-suspended in $1 \mathrm{ml}$ fresh RPMl-1640. The macrophages thus obtained were checked for purity by staining with Wright-Giemsa stain as well as on a flowcytometer (FSC vs SSC; BD Accuri; BD Biosciences, San Jose, CA, USA) and the viability determined using the $0.4 \%$ trypan blue dye exclusion method.

\section{Immunofluorescence studies}

For bacterial trafficking. zSPM isolated from control and infected (mCherry tagged) fish were re-suspended in RPMI-1640 supplemented with 10\% FBS (Gibco/Life Technologies), seeded on ethanol-cleaned glass slides and incubated for $30 \mathrm{~min}$ for their adherence under humid conditions. The adhered ZSPM were sequentially treated with fixation buffer (BD Cytofix; $10 \mathrm{~min}$ ), permeabilization buffer (0.1\% Triton X; $15 \mathrm{~min}$ ), washed twice with PBS and incubated with antibody for early endosomal marker EEA1 (Abcam, Cambridge, UK, 1:500) overnight at $4{ }^{\circ} \mathrm{C}$. The cells were washed with PBS, incubated with secondary antibody (rabbit antimouse IgG-FITC; Santa Cruz Biotechnology Inc., Dallas, TX, USA) for $3 \mathrm{~h}$ at room temperature. The cells were stained with Hoechst $(100 \mu \mathrm{g} / \mathrm{ml}$; $15 \mathrm{~min}$; Sigma-Aldrich Corp.), mounted in fluoroshield (Sigma-Aldrich Corp.) and viewed under a fluorescence microscope $(\times 100$; Zeiss Imager, Z2; Carl Zeiss Iberia, SL, Madrid, Spain).

For TLR4 trafficking. ZSPM were isolated at indicated time points and permeabilized as described above. The cells were then incubated with antibody for TLR4 (rabbit anti-TLR4, GTX113024; GeneTex, Irvine, CA, USA; 1:1000) overnight at $4{ }^{\circ} \mathrm{C}$ followed by incubation with antibody for early endosomal marker EEA1 for $4 \mathrm{~h}$. The cells were washed with PBS, incubated with secondary antibody (goat anti-rabbit lgG- TRITC (Santa Cruz Biotechnology Inc.) for TLR4, 1: 250 and rabbit anti-mouse IgG-FITC for EEA1; 1:250) for $3 \mathrm{~h}$ at room temperature. The cells were washed, stained with Hoechst $(100 \mu \mathrm{g} / \mathrm{ml}$; $15 \mathrm{~min}$; Sigma-Aldrich Corp.), mounted in fluoroshield (Sigma-Aldrich Corp.) and viewed under a fluorescence microscope ( $\times 100$; Zeiss Imager, Z2, Carl Zeiss Iberia).

\section{Statistical analysis}

One-way ANOVA followed by Dunnett post hoc test was performed (SPSS software, Version 13; SPSS Inc., Chicago, IL, USA) to determine statistical significance between control and the experimental groups. Differences were considered significant when $P<0.05$.

\section{ACKNOWLEDGEMENTS}

The work was supported by University of Delhi Doctoral Research Programme (Dean (R)/R\&D/2012/917) and DST-PURSE Grant. NS was supported by Dr. D.S.Kothari Post Doctoral fellowship, UGC (Government of India). AS and MK were supported by fellowships from DBT (Government of India) and CSIR-UGC (Government of India), respectively. We are thankful to Central Instrumentation Facility of Department of Zoology, University of Delhi for providing technical support.

\section{COMPETING INTERESTS}

The authors declare no conflict of interest. 


\section{PUBLISHER'S NOTE}

Springer Nature remains neutral with regard to jurisdictional claims in published maps and institutional affiliations.

\section{REFERENCES}

1 Takeuchi O, Akira S. Pattern recognition receptors and inflammation. Cell 2010; 140: $805-820$.

2 Akira S, Takeda K. Toll like receptor signalling. Nat Rev Immunol 2004; 4: 499-511.

3 Medzhitov R. Toll-like receptors and innate immunity. Nat Rev Immunol 2001; 1: 135-145.

4 Takeda K, Akira S. Toll-like receptors in innate immunity. Int Immunol 2005; 17: 1-14.

5 Siegemund S, Sauer K. Balancing pro- and anti-inflammatory TLR4 signaling. Nat Immunol 2012; 13: 1031-1033.

6 Aksoy E, Taboubi S, Torres D, Delbauve S, Hachani A, Whitehead MA et al. The p110 [delta] isoform of the kinase PI (3) K controls the subcellular compartmentalization of TLR4 signaling and protects from endotoxic shock. Nat Immunol 2012; 13: 1045-1054.

7 Sepulcre MP, Alcaraz-Pérez F, López-Muñoz A, Roca FJ, Meseguer J, Cayuela ML et al. Evolution of lipopolysaccharide (LPS) recognition and signaling: fish TLR4 does not recognize LPS and negatively regulates NF-KB activation. J Immunol 2009; 182: 1836-1845.

8 Su J, Yang C, Xiong F, Wang Y, Zhu Z. Toll-like receptor 4 signaling pathway can be triggered by grass carp reovirus and Aeromonas hydrophila infection in rare minnow Gobiocypris rarus. Fish Shellfish Immunol 2009; 27: 33-39.

9 Palti Y. Toll-like receptors in bony fish: from genomics to function. Dev Comp Immunol 2011; 35: 1263-1272.

10 Huang R, Dong F, Jang S, Liao L, Zhu Z, Wang Y. Isolation and analysis of a novel grass carp toll-like receptor 4 (t|r4) gene cluster involved in the response to grass carp reovirus. Dev Comp Immunol 2012; 38: 383-388.

11 Basu M, Maiti NK, Samanta M. Toll-like receptor (TLR) 4 in mrigal (Cirrhinus mrigala): response to lipopolysaccharide treatment and Aeromonas hydrophila infection. Int Res J Biol Sci 2013; 2: 20-27.

12 Quiniou SM, Boudinot P, Bengtén E. Comprehensive survey and genomic characterization of Toll-like receptors (TLRs) in channel catfish, Ictalurus punctatus: identification of novel fish TLRs. Immunogenetics 2013; 65: 511-530.

13 Zhao F, Li YW, Pan HJ, Shi CB, Luo XC, Li AX et al. Expression profiles of toll-like receptors in channel catfish (Ictalurus punctatus) after infection with Ichthyophthirius multifiliis. Fish Shellfish Immunol 2013; 35: 993-997.

14 Tong C, Lin Y, Zhang C, Shi J, Qi H, Zhao K. Transcriptome-wide identification, molecular evolution and expression analysis of Toll-like receptor family in a Tibet fish Gymnocypris Przewalskii. Fish Shellfish Immunol 2015; 46: 334-345.

15 Swain P, Nayak SK, Nanda PK, Dash S. Biological effects of bacterial lipopolysaccharide (endotoxin) in fish: a review. Fish Shellfish Immunol 2008; 25: 191-201.

16 Pietretti D, Wiegertjes GF. Ligand specificities of Toll-like receptors in fish: indications from infection studies. Dev Comp Immunol 2014; 43: 205-222.

17 Sullivan C, Charette J, Catchen J, Lage CR, Giasson G, Postlethwait JH et al. The gene history of zebrafish t|r4a and t|r4b is predictive of their divergent functions. J Immunol 2009; 183: 5896-5908.

18 Lieschke GJ, Currie PD. Animal models of human disease: zebrafish swim into view. Nat Rev Genet 2007; 8: 353-367.

19 Howe K, Clark MD, Torroja CF, Torrance J, Berthelot C, Muffato M et al. The zebrafish reference genome sequence and its relationship to the human genome. Nature 2013; 496: 498-503.

20 Jault C, Pichon L, Chluba J. Toll-like receptor gene family and TIR-domain adapters in Danio rerio. Mol Immunol 2004; 40: 759-771.

21 Meijer AH, Krens SG, Rodriguez IAM, He S, Bitter W, Snaar-Jagalska BE et al. Expression analysis of the Toll-like receptor and TIR domain adaptor families of zebrafish. Mol Immunol 2004; 40: 773-783.

22 Janda JM, Abbott SL. The genus Aeromonas: taxonomy, pathogenicity, and infection. Clin Microbiol Rev 2010; 23: 35-73.

23 Yardimci B, Yılmaz A. Pathological findings of experimental Aeromonas hydrophila infection in Nile tilapia (Oreochromis niloticus). Ankara Univ Vet Fak Derg 2011; 58: 47-54.

24 Forng RY, Champagne C, Simpson W, Genco CA. Oral microbiology: environmental cues and gene expression in Porphyromonas gingivalis and Actinobacillus actinomycetemcomitans. Oral Dis 2000; 6: 351-365.

25 Saraceni PR, Romero A, Figueras A, Novoa B. Establishment of infection models in Zebrafish larvae (Danio rerio) to study the pathogenesis of Aeromonas hydrophila. Front Microbiol 2016; 7: 1-14.

26 Tiron A, Vasilescu C. Role of the spleen in immunity. Immunologic consequences of splenectomy. Chirurgia (Bucur) 2007; 103: 255-263.
27 Menke AL, Spitsbergen JM, Wolterbeek AP, Woutersen RA. Normal anatomy and histology of the adult zebrafish. Toxicol Pathol 2011; 39: 759-775.

28 Rodríguez I, Novoa B, Figueras A. Immune response of zebrafish (Danio rerio) against a newly isolated bacterial pathogen Aeromonas hydrophila. Fish Shellfish Immunol 2008; 25: 239-249.

29 Reyes-Becerril M, López-Medina T, Ascencio-Valle F, Esteban MÁ. Immune response of gilthead seabream (Sparus aurata) following experimental infection with Aeromonas hydrophila. Fish Shellfish Immunol 2011; 31: 564-570.

30 Basu M, Swain B, Maiti NK, Routray P, Samanta M. Inductive expression of toll-like receptor 5 (TLR5) and associated downstream signaling molecules following ligand exposure and bacterial infection in the Indian major carp, mrigal (Cirrhinus mrigala). Fish Shellish Immunol 2012; 32: 121-131.

31 Banerjee C, Goswami R, Verma G, Datta M, Mazumder S. Aeromonas hydrophila induced head kidney macrophage apoptosis in Clarias batrachus involves the activation of calpain and is caspase-3 mediated. Dev Comp Immunol 2012; 37: 323-333.

32 Banerjee C, Singh A, Das TK, Raman R, Shrivastava A, Mazumder S. Ameliorating ER-stress attenuates Aeromonas hydrophila-induced mitochondrial dysfunctioning and caspase mediated HKM apoptosis in Clarias batrachus. Sci Rep 2014; 4: 5820.

33 Lai R, Liu H, Jakovlić I, Zhan F, Wei J, Yang P et al. Molecular cloning and expression of toll-like receptor 4 (t|r4) in the blunt snout bream (Megalobrama amblycephala). Dev Comp Immunol 2016; 59: 63-76.

34 Pan ZK, Fisher C, Li JD, Jiang Y, Huang S, Chen LY. Bacterial LPS up-regulated TLR3 expression is critical for antiviral response in human monocytes: evidence for negative regulation by CYLD. Int Immunol 2011; 23: 357-364.

35 Zhang XT, Zhang GR, Shi ZC, Yuan YJ, Zheng H, Lin L et al. Expression analysis of nine Toll-like receptors in yellow catfish (Pelteobagrus fulvidraco) responding to Aeromonas hydrophila challenge. Fish Shellfish Immunol 2017; 63: 384-393.

36 Kagan JC, Su T, Horng T, Chow A, Akira S, Medzhitov R. TRAM couples endocytosis of Toll-like receptor 4 to the induction of interferon- $\beta$. Nat Immunol 2008; 9: 361-368.

37 Tanimura N, Saitoh S, Matsumoto F, Akashi-Takamura S, Miyake K. Roles for LPSdependent interaction and relocation of TLR4 and TRAM in TRIF-signaling. Biochem Biophys Res Commun 2008; 368: 94-99.

38 Watanabe S, Kumazawa Y, Inoue J. Liposomal lipopolysaccharide initiates TRIFdependent signaling pathway independent of CD14. PLoS One 2013; 8: e60078.

39 Kumagai Y, Takeuchi O, Akira S. TLR9 as a key receptor for the recognition of DNA. Adv Drug Del Rev 2008; 60: 795-804.

40 Newton K, Dixit VM. Signaling in innate immunity and inflammation. Cold Spring Harb Perspect Biol 2012; 4: a006049.

41 Kaiser WJ, Upton JW, Mocarski ES. Receptor-interacting protein homotypic interaction motif-dependent control of NF-KB activation via the DNA-dependent activator of IFN regulatory factors. J Immunol 2008; 181: 6427-6434.

42 Sato S, Sugiyama M, Yamamoto M, Watanabe Y, Kawai T, Takeda K et al. Toll/IL-1 receptor domain-containing adaptor inducing IFN- $\beta$ (TRIF) associates with TNF receptor-associated factor 6 and TANK-binding kinase 1, and activates two distinct transcription factors, NF-KB and IFN-regulatory factor-3, in the Toll-like receptor signaling. J Immunol 2003; 171: 4304-4310.

43 Kawagoe T, Sato S, Jung A, Yamamoto M, Matsui K, Kato H et al. Essential role of IRAK-4 protein and its kinase activity in Toll-like receptor-mediated immune responses but not in TCR signaling. J Exp Med 2007; 204: 1013-1024.

44 Phelan PE, Mellon MT, Kim CH. Functional characterization of full-length TLR3, IRAK-4, and TRAF6 in zebrafish (Danio rerio). Mol Immunol 2005; 42: 1057-1071.

45 Umasuthan N, Bathige SDNK, Whang I, Lim BS, Choi CY, Lee J. Insights into molecular profiles and genomic evolution of an IRAK4 homolog from rock bream (Oplegnathus fasciatus): immunogen-and pathogen-induced transcriptional expression. Fish Shellfish Immunol 2015; 43: 436-448.

46 Häcker $\mathrm{H}$, Tseng PH, Karin M. Expanding TRAF function: TRAF3 as a tri-faced immune regulator. Nat Rev Immunol 2011; 11: 457.

47 Kovarik P, Castiglia V, Ivin M, Ebner F. Type 1 interferons in bacterial infections: a balancing act. Front Immunol 2016; 7: 1-8.

48 Kawai T, Akira S. Signaling to NF-KB by Toll-like receptors. Trends Mol Med 2007; 13: $460-469$.

49 Cusson-Hermance N, Khurana S, Lee TH, Fitzgerald KA, Kelliher MA. Rip1 mediates the Trif-dependent toll-like receptor 3- and 4-induced NF-KB activation but does not contribute to interferon regulatory factor 3 activation. $J$ Biol Chem 2005; 280: 36560-36566.

50 Ofengeim D, Yuan J. Regulation of RIP1 kinase signalling at the crossroads of inflammation and cell death. Nat Rev Mol Cell Biol 2013; 14: 727-736.

51 Lawrence T. The nuclear factor NF-KB pathway in inflammation. Cold Spring Harb Perspect Biol 2009; 1: a001651.

52 Thanawongnuwech R, Thacker B, Halbur P, Thacker EL. Increased production of proinflammatory cytokines following infection with porcine reproductive and respiratory syndrome virus and Mycoplasma hyopneumoniae. Clin Diagn Lab Immunol 2004; 11: 901-908. 
53 Cyktor JC, Turner J. Interleukin-10 and immunity against prokaryotic and eukaryotic intracellular pathogens. Infect Immun 2011; 79: 2964-2973.

54 Gudbrandsdottir S, Hasselbalch HC, Nielsen CH. Activated platelets enhance IL-10 secretion and reduce TNF-a secretion by monocytes. J Immunol 2013; 191: 4059-4067.

55 Arpaia N, Barton GM. The impact of Toll-like receptors on bacterial virulence strategies. Curr Opin Microbiol 2013; 16: 17-22.

56 Gruenberg J, Van der Goot FG. Mechanisms of pathogen entry through the endosomal compartments. Nat Rev Mol Cell Biol 2006; 7: 495.

57 Mitchell G, Chen C, Portnoy DA. Strategies used by bacteria to grow in macrophages. Microbiol Spectr 2016; 4: 1-38.

58 Vanlandingham PA, Ceresa BP. Rab7 regulates late endocytic trafficking downstream of multivesicular body biogenesis and cargo sequestration. $J$ Biol Chem 2009; 284: 12110-12124.

59 Fritz JH, Girardin SE, Fitting C, Werts C, Mengin-Lecreulx D, Caroff M et al. Synergistic stimulation of human monocytes and dendritic cells by Toll-like receptor 4 and NOD1-and NOD2-activating agonists. Eur J Immunol 2005; 35: 2459-2470.

60 Li M, Wang QL, Lu Y, Chen SL, Li Q, Sha ZX. Expression profiles of NODs in channel catfish (Ictalurus punctatus) after infection with Edwardsiella tarda, Aeromonas hydrophila, Streptococcus iniae and channel catfish hemorrhage reovirus. Fish Shellfish Immunol 2012; 33: 1033-1041.

61 Pauleau AL, Murray PJ. Role of nod2 in the response of macrophages to toll-like receptor agonists. Mol Cell Biol 2003; 23: 7531-7539.

62 Wilson JM, Bunte RM, Carty AJ. Evaluation of rapid cooling and tricaine methanesulfonate (MS222) as methods of euthanasia in zebrafish (Danio rerio). J Am Assoc Lab Anim Sci 2009; 48: 785-789.

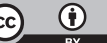

This work is licensed under a Creative Commons Attribution 4.0 International License. The images or other third party material in this article are included in the article's Creative Commons license, unless indicated otherwise in the credit line; if the material is not included under the Creative Commons license, users will need to obtain permission from the license holder to reproduce the material. To view a copy of this license, visit http://creativecommons.org/licenses/ by/4.0/

(c) The Author(s) 2017 